\title{
Robust Optimization of Multi-link Suspension Compliance Characteristics Based on Taguchi Method
}

\author{
Shuhua Liao", a, Kaibin Cao1, b, Hongfa Wei², Jinwu Liu², Cuihua Xie² \\ ${ }^{1}$ College of Automobile and Transportation, Guangxi University of Science and Technology.530000, \\ China; \\ ²Liuzhou KH Automotive Technologies Co. Ltd, Liuzhou 545006, China. \\ aliaohsh60@163.com, b501333069@qq.com
}

Keywords: Bushing stiffness, Compliance Characteristics, Taguchi Method, AHP, Robustness.

\begin{abstract}
Point to problems of long tuning cycle and unapparent performance improvement in the chassis tuning process of keep changing suspension bushing parameters, considering the error of suspension installation, the robust optimization based on Taguchi method is proposed. Use Adams software to model suspension and by $\mathrm{K} \& \mathrm{C}$ test the accuracy of suspension. Utilize Isight software to analyze the degree of influence of bushing stiffness on suspension compliance characteristics. Adopt the Analytic Hierarchy Process method on the uniformity of objective function of suspension compliance characteristics. Design orthogonal experiment based on Taguchi Method to achieve suspension Pareto solution set. According to the best controllable factor, verify the optimization results by the Monte Carlo method. The result illustrated that both the suspension and robustness improved a lot after optimization.
\end{abstract}

\section{Introduction}

Suspension Compliance Characteristics means when tires get the lateral force, longitudinal force and aligning torque, the wheel alignment parameters arise from the elastic deformation of bushings would change with these force and torque $[1,2]$. Most of the suspension of vehicles uses bushings now. Suspension compliance characteristics have important influences on the vehicle's handling stability [3]. In the chassis tuning of sample cars, engineers used to ensure the invariable of sample cars suspension hard points as their experience, by borrowing parts, keeping altering suspension bushing stiffness parameters to improve vehicle's handling stability [4]. It is hard for engineers to make indexes in a comparable perfect condition for vehicles with many $\mathrm{H}$-arm multi-link rear suspension and reciprocal bond of parameters. Also it takes longer time to tuning. Therefore, utilizing Taguchi Method to optimize multi-objective robustness of $\mathrm{H}$-arm multi-link rear suspension will reach multi indexes perfect condition and shorten the tuning time, which can ensure the research and development process.

\section{The Establishment of H-Arm Suspension Modeling}

\subsection{Suspension Structure Analysis.}

A vehicle's H-arm multi-link rear suspension is taken as sample to analyze. Its suspension system consists of toe control arm, camber control arm, H-control arm, spring, damper and upright. By Adams software, H-arm multi-link suspension simulation model is established, Fig. 1. In the model, both the hard points and bushing stiffness use prototype measured values. Fx and Fy mean the Radial stiffness. Fz means axial stiffness. Tx and Ty means radical torsional stiffness. Tz means axial torsional stiffness. In fig. 1, the influence on Suspension Compliance Characteristics of toe control arm bushing 1, 2, camber control arm bushing 3, 4, H-control arm bushing 5-8, are showed. 


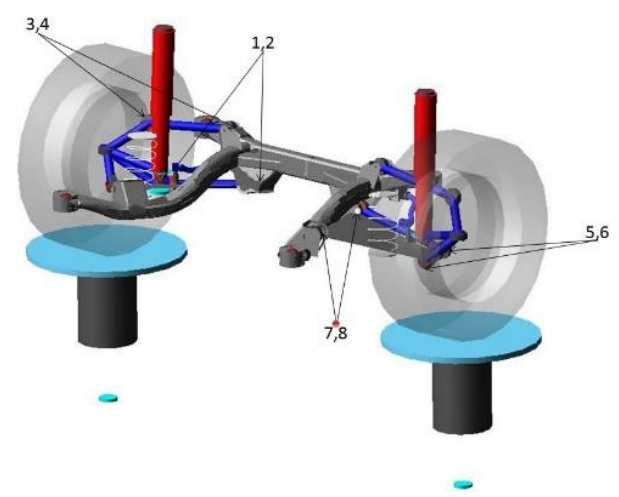

Fig. 1 Model of H-Arm Suspension

\subsection{Simulation Model and Experimental Verification.}

By suspension $\mathrm{K} \& \mathrm{C}$ Test to sample cars, compare experiment results with simulation results to test the accuracy of the model. Fig. 2 and 3 are under the Lateral force condition, the change of Toe Angle and Wheel Travel Track. There is a good correlation between the experiment results and simulation results. To contrastively analyze the other condition of suspension, they all have satisfactory accuracy.

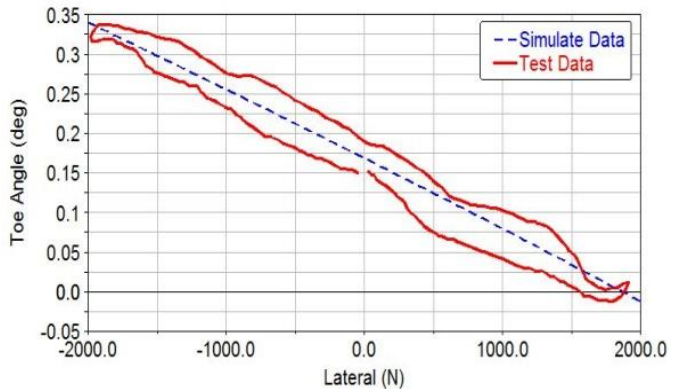

Fig. 2 Toe Angle Rate

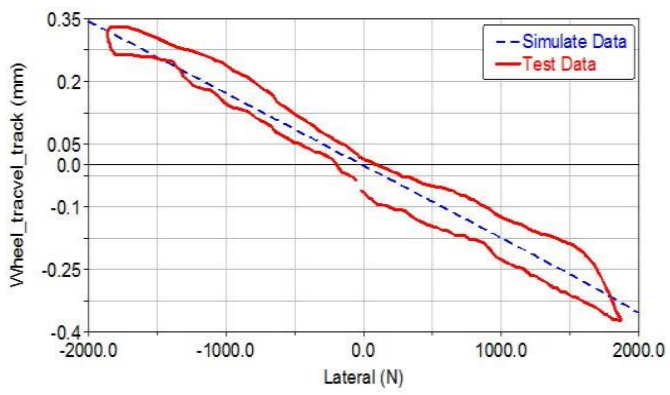

Fig. 3 Wheel Travel Track Rate

\section{Taguchi Robustness Design Theory}

The robustness of product quality refers to the ability of resisting uncertain interference factors, or the characteristics of insensibility to design parameter and noise that change within limits $[5,6]$. When there is deviation, there is loss of quality. Y is put as product quality characteristic value, and target value $y_{0}$. $\mathrm{K}$ is the coefficient of loss of quality.

The function for loss of quality is:

$$
\mathrm{L}(\mathrm{y})=\mathrm{K}\left(\mathrm{y}-\mathrm{y}_{0}\right)
$$

As y has its randomness, proposing that $\mathrm{y}$ is normally distributed between $\mathrm{y} \sim \mathrm{N}\left(\mu, \sigma^{2}\right) . \mu$ is the mean value of quality coefficient. The product quality is valued by average quality loss function $\mathrm{E}$ $\{\mathrm{L}(\mathrm{y})\}$ :

$\mathrm{E}\{\mathrm{L}(\mathrm{y})\}=\mathrm{E}[\mathrm{K}(\mathrm{y}-\mathrm{y} 0) 2]=\mathrm{K}(\sigma 2+\delta 2)$

$\sigma^{2}$ is the variance of quality coefficient; $\delta$ is the absolute deviation quality coefficient.

Robust Design Optimization requires not only mean value be the smallest of target value $\delta$, at the same time, Functional fluctuations $\sigma^{2}$, arise by all kinds of interference factors, need to be as small as possible. Taguchi Method uses the value of Signal-Noise ratio to measure product robustness, and combines the controllable factor and noise factor by orthogonal experiment design to find the best parameter combination to increase the robustness of product quality.

For the elastic kinematics of the suspension characteristics, the requirement of qualitative characteristics: no negative, as small as possible, zero the target value. It is called smaller the better feature. The average quality loss function is:

$$
\mathrm{E}\{\mathrm{L}(\mathrm{y})\}=\frac{1}{n} \sum_{i=1}^{n} y_{i}^{2}
$$

Signal-Noise ratio is: 
$\mathrm{SRN}=-10 \lg \left(\frac{1}{n} \sum_{i=1}^{\mathrm{m}} y_{i}^{2}\right)$

The larger Signal-Noise ratio, the smaller loss of quality, the better the quality is.

\section{Sensitivity analysis}

The sensitivity Analysis of bushing stiffness is through co-simulation of Isight and Adams softwares. Utilizing Optimal Latin hypercube to design experiment and find remarkable elements of suspension compliance characteristics in Isight software. Taking bushing stiffness as variable, parallel aligning torque, parallel lateral force and parallel longitudinal force, all three operating condition to analyze suspension compliance characteristics. The mean value of slopes of Toe Angle-Aligning Torque, Camber Angle-Lateral Force, Toe Angle-Lateral Force, Wheel Travel Track-Lateral Force, Wheel Travel Base-Longitudinal Force and Toe Angle-Longitudinal Force are objective responses. As there are more bushings in $\mathrm{H}$-arm suspension, 12 parameters from 8 bushings of 48 parameters are preliminarily selected, which have a great influence on suspension compliance characteristics. Sensitivity analysis is done to 12 parameters. Table 1 takes coefficient variation of bushing stiffness as independent variable, which is 1 initially, variation range is $(0.5,2)$.

Record:

T1=H_aligningtorque_toeratemean;

T2=H_lateralforce_camberratemean T3=H_lateralforce_toeratemean: T4=H_lateralforce_trackratemean; T5=H_ longitudinalforce_ baseratemean; $\mathrm{T} 6=\mathrm{H} \_$longitudinalforce_toeratemean;

Table .1 Suspension Compliance Characteristics Sensitivity Analysis Results (\%)

\begin{tabular}{|c|c|c|c|c|c|c|c|}
\hline Variable & Numble & T1 & T2 & T3 & T4 & T5 & T6 \\
\hline camber_ctl_arm_inner_fy & 2 & 0.51 & 23.36 & 4.42 & -21.33 & -1.75 & -1.80 \\
\hline camber_ctl_arm_outer_fy & 2 & 0.23 & 24.24 & 3.74 & -21.45 & -1.63 & -1.65 \\
\hline h_arm_front_inner_f & 4 & 12.47 & 0.10 & 9.35 & 6.59 & 39.70 & -29.88 \\
\hline h_arm_front_outer_fx & 1 & -0.06 & 0.66 & -0.67 & -0.22 & -10.84 & 2.42 \\
\hline h_arm_frontouter_fy & 4 & 27.37 & 0.56 & 15.02 & 9.16 & 7.06 & -19.10 \\
\hline h_arm_front_outer_fz & 5 & 19.86 & 5.90 & 21.27 & 14.42 & 10.69 & 16.41 \\
\hline h_arm_rear_inner_fy & 1 & 0.57 & 10.86 & 4.33 & 8.52 & -1.59 & -0.01 \\
\hline h_arm_rear_outer_fx & 2 & 12.24 & 4.49 & -8.58 & -2.89 & 0.70 & 2.50 \\
\hline h_arm_rear_outer_fy & 1 & 2.63 & -0.80 & -0.18 & 0.89 & -12.73 & 9.41 \\
\hline h_arm_rear_outer_fz & 4 & 16.06 & 3.75 & 15.16 & 9.62 & 7.99 & 12.47 \\
\hline toe_ctl_arm_inner_fy & 2 & 4.60 & 13.62 & -10.37 & 2.34 & 1.72 & -2.04 \\
\hline toe_ctl_arm_outer_fz & 1 & 3.40 & 11.65 & -6.92 & 2.57 & 3.59 & -2.32 \\
\hline Cond
\end{tabular}

Considering each variable in sensitivity analysis results, camber_ctl_arm_inner_fy (F1), camber_ctl_arm_outer_fy (F2), h_arm_front_inner_fx (F3), h_arm_front_outer_fy (F4), h_arm_front_outer_fz (F5), h_arm_rear_outer_fx (F6), h_arm_rear_outer_fz (F7) and toe_ctl_arm_inner_fy (F8) are put as controllable factors. Because of the error of suspension installation hard points, camber_ctl_arm_inner_y, damper_lower_z, damper_upper_z and toe_ctl_arm_inner_y are taken as noise factors, and the error changes between plus or minus $1 \mathrm{~mm}$ based on the original hard point coordinates.

\section{Determining objective Function}

Considering competing vehicle database and determining the ideal range of this $\mathrm{H}$-arm suspension response compliance characteristics as Table 2.

Table .2 Response Compliance Characteristics Ideal Range

\begin{tabular}{|c|c|c|c|c|c|c|}
\hline Response & OB 1 & OB 2 & OB 3 & OB 4 & OB 5 & OB 6 \\
\hline \multirow{2}{*}{ Ideal Range } & $-6 \mathrm{E}-7 \pm$ & $-2.4 \mathrm{E}-4 \pm$ & $-3.6 \mathrm{E}-5 \pm$ & $-1 \mathrm{E}-4 \pm$ & $-1.2 \mathrm{E}-3 \pm$ & $-1 \mathrm{E}-5 \pm$ \\
& $60 \%$ & $10 \%$ & $30 \%$ & $30 \%$ & $10 \%$ & $60 \%$ \\
\hline
\end{tabular}

The evaluation index of Suspension Compliance Characteristics:

$(i=1,2,3,4,5,6)$ 


$$
C i=\left|\frac{T i-O B i}{O B i}\right|
$$

$\mathrm{OB} i$ is ideal value of quantized response characteristics, $T i$ is system real-time output response characteristics.

Combine with engineering experience, AHP compare 6 characteristics by 1-9 scale two-two comparison. Build judgments matrix, calculate the weight of each response to suspension compliance characteristics and acquire the largest eigenvalue $\lambda_{\max }$ by judgment matrix. In Tab. 3 can find average random consistency index R.I [7], Tab. 4, calculating the consistency index C.I $=\left(\lambda_{\max }-\mathrm{n}\right) /(\mathrm{n}-1)$ and the consistency ratio C.R=C.I/R.I to estimate feasibility analysis of matrix. It is acceptable if C.R $<0.1$ that judge matrix is consistent. While if $C . R>0.1$, judge matrix need to be amended.

Table $.31-9$ order positive and negative matrix calculation 1000 times the average random consistency index R.I.

\begin{tabular}{|c|c|c|c|c|c|c|c|c|c|}
\hline Matrix Order & 1 & 2 & 3 & 4 & 5 & 6 & 7 & 8 & 9 \\
\hline R.I & 0 & 0 & 0.52 & 0.89 & 1.12 & 1.26 & 1.36 & 1.41 & 1.46 \\
\hline
\end{tabular}

Table 4 Pairwise comparison matrix and consistency check

\begin{tabular}{|c|c|c|c|c|c|c|c|}
\hline $\mathrm{C}$ & $\mathrm{C} 1$ & $\mathrm{C} 2$ & C3 & C4 & C5 & C6 & W \\
\hline $\mathrm{C} 1$ & 1 & $1 / 3$ & $1 / 3$ & $1 / 2$ & $1 / 3$ & 2 & $8.06 \%$ \\
\hline $\mathrm{C} 2$ & 3 & 1 & 2 & 4 & 3 & 4 & $35.78 \%$ \\
\hline $\mathrm{C} 3$ & 3 & $1 / 2$ & 1 & 2 & 2 & 4 & $23.02 \%$ \\
\hline $\mathrm{C} 4$ & 2 & $1 / 4$ & $1 / 2$ & 1 & $1 / 2$ & 2 & $10.82 \%$ \\
\hline $\mathrm{C} 5$ & 3 & $1 / 3$ & $1 / 2$ & 2 & 1 & 3 & $16.61 \%$ \\
\hline C6 & $1 / 2$ & $1 / 4$ & $1 / 4$ & $1 / 2$ & $1 / 3$ & 1 & $5.70 \%$ \\
\hline \multicolumn{8}{|c|}{$\lambda \max =6.2024 ; \mathrm{C} . \mathrm{I}=0.0405 ; \mathrm{C} . \mathrm{R}=0.0321<0.1$} \\
\hline
\end{tabular}

The characteristics of objective function of elastic kinematics of the suspension:

$C=\sum_{i=1}^{n} W i *\left|\frac{T i-O B i}{O B i}\right|$

According to engineering $\mathrm{K} \& \mathrm{C}$ data analysis, the smaller objective function response, the better it is.

\section{Robust Design optimization Based on Taguchi Method}

As the controllable factor and noise factor are fixed, the interactions between parameters are not taken into consideration, orthogonal experiment design is conducted. The experiment of controllable factor utilized the orthogonal array $L_{32}\left(3^{8}\right)$, while noise factor utilized orthogonal array $L_{8}\left(2^{4}\right)$. The total number of test is 256 . As the factor of the first Taguchi experiment may be far from the most optimum value, which cannot meet the need of precision. In order to improve the range of the factors precision, the second time Taguchi factor choose to base on the result of the first Taguchi analysis, and do experimental analysis again. The fig. 4, Pareto solution set of the experiment design are acquired.

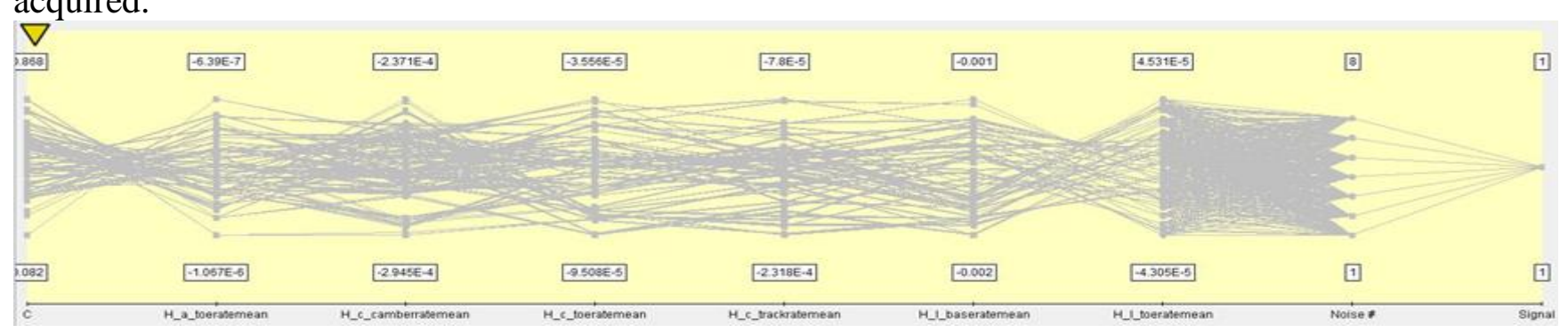

Fig. 4 Experimental design optimization Pareto solution set

Fig.4,under three conditions of parallel aligning torque, parallel lateral force and parallel Longitudinal force, The mean value of Toe Angle-Aligning Torque slope range is [-1.067E-6, 6.39E-7]. The mean value of Camber Angle-Lateral Force slope range is [-2.945E-4, -2.371E-4]. The mean value of Toe Angle-Lateral Force slope range is [-9.508E-5, -3.556E-5]. The mean value of 
Wheel Travel Track-Lateral Force slope range is [-2.318E-4, -7.8E-5]. The mean value of Wheel Travel Base-Longitudinal Force slope range is [-0.002, -0.001]. The mean value of Toe Angle-Longitudinal Force slope range is [-4.305E-5, 4.531E-5]. Compared with figure 1, the Pareto disaggregation is all covered in the ideal range of response characteristics.

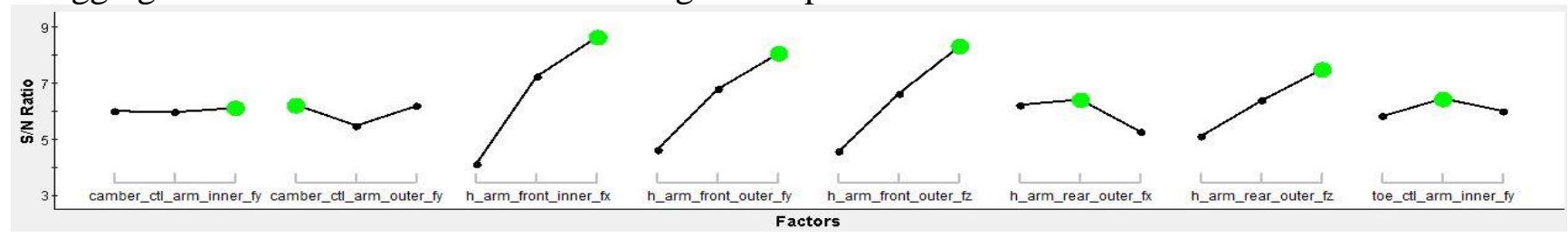

Fig. 5 Signal-Noise ratio main effect graph

Fig. 5 is Signal-Noise ratio main effect graph. The bigger Signal-Noise ratio value is, the better the suspension robustness is, vice versa. We select the optimal portfolio of controllable factors Tab.5.

Table 5 the optimal portfolio of controllable factors

\begin{tabular}{|c|c|c|c|c|c|c|c|c|}
\hline Controllable Factor & F1 & F2 & F3 & F4 & F5 & F6 & F7 & F8 \\
\hline Level & 2 & 1 & 2 & 2 & 2 & 1.5 & 2 & 1.5 \\
\hline
\end{tabular}

\section{Analysis and Verification of The Results}

The result of robust optimization compares with the initial value of suspension compliance characteristics. From Tab. 6, under the conditions of parallel aligning torque, parallel lateral force and parallel longitudinal force, the mean value of changing Toe angle, Camber angle, Wheel Travel Track, and Wheel Travel Base are within the target range, which means there is a big improvement on the characteristics of the elastic kinematics of the suspension.

Table 6 the result of optimization

\begin{tabular}{|c|c|c|c|}
\hline Optimal Object & Before & After & Target field \\
\hline T1 & $-1.065 \mathrm{E}-06$ & $-6.399 \mathrm{E}-07$ & $-6 \mathrm{E}-07 \pm 60 \%$ \\
\hline T2 & $-2.936 \mathrm{E}-04$ & $-2.521 \mathrm{E}-04$ & $-2.4 \mathrm{E}-04 \pm 10 \%$ \\
\hline T3 & $-7.206 \mathrm{E}-05$ & $-3.672 \mathrm{E}-05$ & $-3.6 \mathrm{E}-05 \pm 30 \%$ \\
\hline T4 & $-1.745 \mathrm{E}-04$ & $-1.053 \mathrm{E}-04$ & $-1 \mathrm{E}-04 \pm 30 \%$ \\
\hline T5 & $-1.837 \mathrm{E}-03$ & $-1.190 \mathrm{E}-03$ & $-1.2 \mathrm{E}-03 \pm 10 \%$ \\
\hline T6 & $1.404 \mathrm{E}-05$ & $-8.969 \mathrm{E}-06$ & $-1 \mathrm{E}-05 \pm 60 \%$ \\
\hline
\end{tabular}

Utilized simple random sampling method of the Monte Carlo for the before and after optimization to prove the validity of optimization. 1000 sample points are selected, Fig. 6. Considering the error of suspension installation hard points, the each response variance continues to be smaller, which means the robustness of suspension of after optimization improved a lot and the effectiveness is optimized.

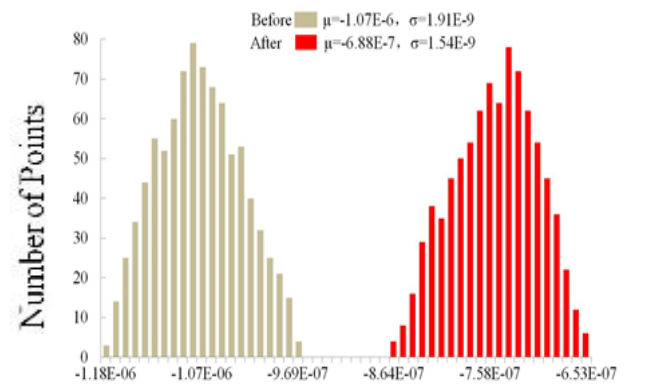

H_Aligningtorque_Toeratemean $\left(\mathrm{deg} / \mathrm{N}^{*} \mathrm{~m}\right)$

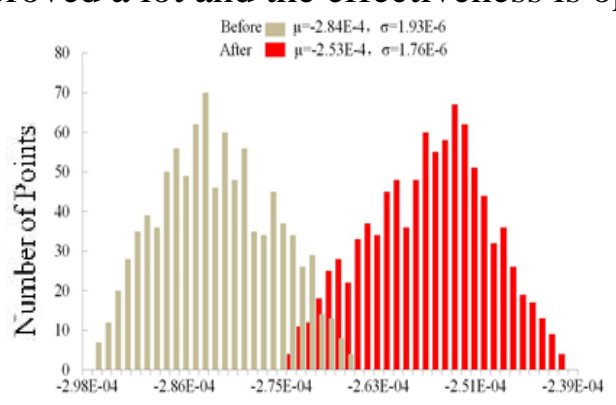

H_lateralforce_camberratemean $(\mathrm{deg} / \mathrm{N})$ 


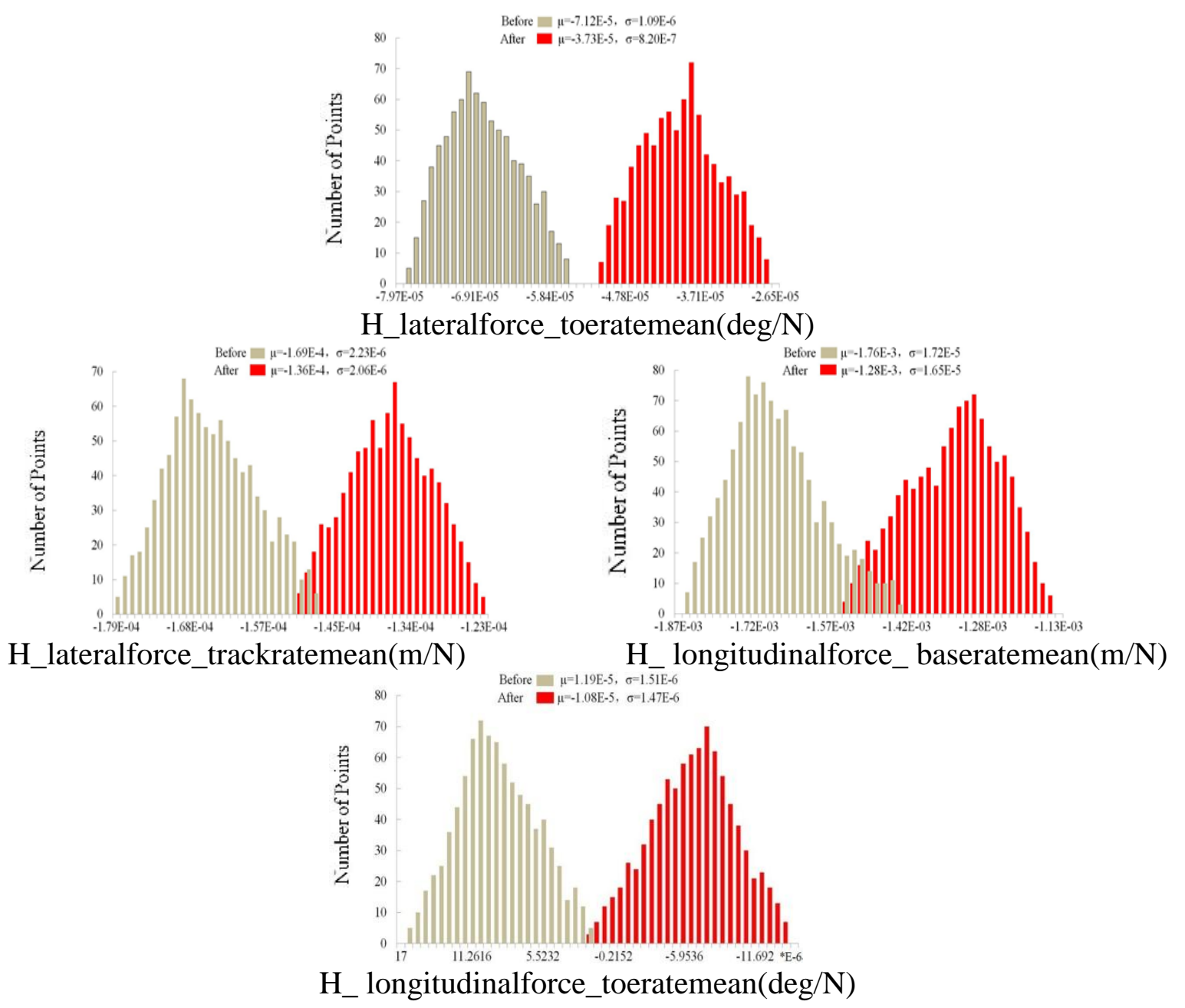

Fig. 6 the probability distribution of the target before and after optimization

\section{Conclusion}

Utilized Taguchi method to design Robust Optimization of H-arm multi-link suspension, and used AHP to quantize subjective factors by engineering evaluation. Also, uniformity of objective response is achieved. The optimization reached target suspension characteristics range, which means the effectiveness of the method and the robustness of suspension are improved.

By co-simulation method of Isight and Adams to tune sample cars, whose performance are optimized and the most optimal portfolio are selected. Compared with previous method of adjusting the bushing stiffness, it shortened the development cycle, raised working efficiency, and provided analysis of complete cars performance.

\section{References}

[1]. Kang J S, Yun J R, Lee J M. Elasto-kinematic Analysis and Optimization of Suspension Compliance Characteristics. SAE Paper 970104.

[2]. Gao Jin, Yang Xiujian. Sensitivity Study of Bushing Stiffness Affecting Suspension Compliance Characteristics and Transient Handling Stability [J]. Automobile Technology.2013. (11).

[3]. Peng Yaqi, Xiong Rui. Effects of bushing stiffness to the compliance characteristics of multi-link suspension [J]. Modern Manufacturing Engineering.2015. (10).

[4]. W. C. Mitchell, Suspension Geometry: Theory vs. K\&C Measurement, SAE Paper, and 2008-01-2948. 
[5]. Zang C, Friswell M I, Mottershead J E. A review of robust optimal design and its application in dynamics [J]. Computers and Structures, 2005, 83 (4/5):315-326.

[6]. Ying Zhang. Robust Design Optimization of the Automotive Suspension Based on Taguchi Method [J].Machinery\&Electronics.2015. (9).

[7]. Guopu Peng. AHP in Evaluating Government Performance: Determining Indicator Weight [J]. China Soft Science.2004. (6).136-139. 